\title{
Norois
}

Environnement, aménagement, société

\section{Tempête Xynthia à la Faute-sur-Mer : une analyse $a$ posteriori de l'impact des « zones noires » et des alternatives possibles}

Storm Xynthia at La Faute-sur-Mer: an ex-ante analysis of consequences of resettlement and alternative scenarios

\section{Axel Creach}

\section{OpenEdition}

\section{Journals}

Édition électronique

URL : https://journals.openedition.org/norois/9168

DOI : $10.4000 /$ norois. 9168

ISSN : $1760-8546$

\section{Éditeur}

Presses universitaires de Rennes

\section{Édition imprimée}

Date de publication : 22 novembre 2019

Pagination : 43-63

ISBN : 978-2-7535-7875-3

ISSN : 0029-182X

\section{Référence électronique}

Axel Creach, « Tempête Xynthia à la Faute-sur-Mer : une analyse a posteriori de l'impact des « zones noires » et des alternatives possibles », Norois [En ligne], 251 | 2019, mis en ligne le 02 janvier 2022, consulté le 08 janvier 2022. URL : http://journals.openedition.org/norois/9168 ; DOI : https://doi.org/ $10.4000 /$ norois. 9168 


Presses
Universitaires
de Rennes

\title{
Tempête Xynthia à La Faute-sur-Mer : une analyse a posteriori de l'impact des «zones noires » et des alternatives possibles
}

\author{
Storm Xynthia at La Faute-sur-Mer: An Ex-ante Analysis of Consequences \\ of Resettlement and Alternative Scenarios
}

\author{
Axel CReach
}

UMR 8591 LGP, CNRS, Institut de Géographie Faculté des Lettres, Sorbonne Université, 191, rue Saint-Jacques,

75005 PARIs. (axel.creach@sorbonne-universite.fr)

Résumé : En février 2010, la commune de La Faute-sur-Mer (Vendée) est durement impactée par la tempête Xynthia. 29 personnes décèdent (pour une population de 1008 habitants), piégées à l'intérieur de chez elles. Face à ce constat, l'État décide le rachat et la destruction des constructions jugées les plus dangereuses entrainant la disparition de 552 maisons (sur 3737 habitations). La commune est ainsi doublement touchée par Xynthia. La Cour des Comptes se montre critique quant aux sommes engagées pour cette mesure qu'elle qualifie d'« une utilisation inefficace des fonds publics. » En utilisant la méthodologie de l'indice VIE, cette recherche montre que des alternatives étaient possibles permettant à la fois de réduire efficacement la vulnérabilité des constructions pour leurs occupants, maintenir un maximum de logements sur la commune, tout en maittrisant les dépenses publiques. Ainsi, envisager simultanément un renforcement de la prévention, l'adaptation architecturale des constructions et la relocalisation auraient permis de supprimer l'intégralité des constructions dangereuses pour un coût deux fois inférieur (76 millions contre 160 pour les « zones noires ») tout en maintenant $70 \%$ des constructions détruites après Xynthia.

\begin{abstract}
In February 2010, La Faute-sur-Mer municipality (Vendée) was hit by Storm Xynthia. 29 deaths occurred (over a population of 1,008 inhabitants), all recorded inside residential houses where people were trapped. French government decided to buy and destruct houses which were considered as too dangerous and the municipality lost 552 houses (over 3,737). This policy was criticized for its high cost as well as it impacted the municipality for long term. Using VIE index methodology, this research aims to highlight that alternative scenarios were possible in order to improve efficiency to protect human lives, reduce costs and maintain people. Applying simultaneously prevention, architectural adaptation and resettlement would have offered a better result to reduce building's vulnerability for people for a cost two time lower and allowing to maintain $70 \%$ of buildings destroyed after the Storm.
\end{abstract}

Mots clés : risque - vulnérabilité - Xynthia - aménagement littoral - adaptation - recul stratégique

Keywords: risk - vulnerability - Xynthia - coastal management - adaptation - resettlement 


\section{INTRODUCTION}

En février 2010, la tempête Xynthia a touché les côtes atlantiques françaises. Cette tempête, modérée à bien des égards (dépression, vitesse du vent), s'est conjuguée à une marée haute de coefficient 102, entraînant des niveaux marins particulièrement élevés (Feuillet et al., 2012). Elle a atteint son paroxysme au droit des côtes des régions Pays de la Loire et Poitou-Charentes. Ces deux régions se caractérisent par un littoral bas et comptent à elles deux $44 \%$ des zones potentiellement inondables de France métropolitaine pour un événement équivalent à Xynthia (CETMEF et al., 2009).

Ainsi, sur les 134 communes littorales de ces deux régions, 110 ont été inondées lors de Xynthia, dont 53 ont vu une partie de leurs zones urbanisées submergées (Devaux et al., 2012; SOGREAH, 2010).

L'inondation de lotissements résidentiels, couplée à d'autres facteurs comme le moment de survenue de la submersion (de nuit), l'émission de consignes de confinement ayant pu entrer en contradiction avec l'inondation des logements (Kolen et al., 2013; Vinet et al., 2011) ou l'âge de la population touchée (Vinet et al., 2011) ont entraîné le décès par noyade de 41 personnes, piégées chez elles par la montée de l'eau (Vinet et al., 2011). Cela fait de Xynthia l'événement de submersion marine le plus meurtrier en France du dernier siècle (Boudou, 2015) et un événement particulier parmi les événements d'inondations à l'échelle mondiale pour la surreprésentation des décès à l'intérieur de bâtiments $(100 \%$ contre $6 \%$ en moyenne d'après Jonkman et Kelman, 2005).

Cela pose la question sous-jacente et soulevée de longue date (Chauvet et Renard, 1978) de l'urbanisation littorale comme facteur d'exposition au risque de submersion marine (Chauveau et al., 2011 ; Vinet et al., 2012).

Ainsi, à elle seule, la commune de La Faute-surMer a compté 29 victimes, soit $70 \%$ des décès par noyade liés à l'événement Xynthia (Vinet et al., 2011 ) et $3 \%$ de sa population de 2006 (1 008 habitants en 2006 d'après l'INSEE). Elle est rapidement devenue le symbole et le « cas d'école » illustrant les conséquences d'une urbanisation importante des communes littorales ayant fait fi du risque de submersion marine. À titre d'exemple, sur les sept communes ayant enregistré des décès lors de Xynthia, quatre d'entre-elles avaient connu une augmenta- tion de leur population supérieure à la moyenne nationale (+38\% au national contre $+66 \%$ à Aytré, +180\% à La Flotte-en-Ré) sur la période 1968-2007 (Vinet et al., 2012). Sur la même période, hormis Xynthia, aucune submersion marine majeure ne s'était produite en France métropolitaine, ce qui ne veut pas dire que cet aléa était inconnu comme l'ont justement signalé Chauvet et Renard (1978) pour les côtes vendéennes.

Ainsi, les conséquences de l'événement Xynthia ne sont pas tant celles de la submersion que des choix d'aménagement qui ont été faits dans les décennies précédentes sur le territoire. Ce constat peut d'ailleurs être étendu à d'autres catastrophes récentes comme l'ouragan Katrina qui a frappé La Nouvelle-Orléans en 2005 (Burby, 2006), ou le tsunami à l'origine de la catastrophe de Fukushima, en 2011, au Japon (Funabashi, 2012).

À la suite de ces choix d'aménagement et notamment la mise en avant de la dangerosité que pouvaient représenter les constructions résidentielles pour leurs occupants (Creach et al., 2015; Vinet et al., 2012; Vinet et al., 2011), l'État a décidé une déconstruction des maisons considérées comme trop dangereuses pour y vivre. Cette mesure, connue sous le vocable «zones noires » ou « zones de solidarité » (Mercier et Chadenas, 2012), a entraîné le rachat et la destruction de 1628 constructions résidentielles dont 552 sur la commune de La Faute-sur-Mer (Cour des Comptes, 2012) soit près de $15 \%$ de son parc immobilier (estimé à 3737 résidences en 2006 d'après l'INSEE).

Déjà endeuillée, la commune de La Faute-surMer a été doublement marquée par l'événement à la suite de l'action de l'État qui a été critiquée pour sa mise œuvre précipitée et son manque de transparence (Mercier et Chadenas, 2012; Verger, 2010). Ainsi, la Cour des Comptes a pointé sa cherté parlant même d'une " utilisation inefficace des fonds publics » (Cour des Comptes, 2012). De plus, les destructions ont entraîné le départ d'habitants tandis que la médiatisation de La Faute-sur-Mer aurait entraîné une baisse de la fréquentation touristique, ces deux tendances ayant des conséquences à plus long terme sur la commune.

Il est donc légitime de s'interroger a posteriori sur les conséquences directes et indirectes de l'événement Xynthia sur la commune de La Faute-sur-Mer, compte tenu de l'ampleur du bilan humain et des 
déconstructions pour une commune de petite taille. Les conséquences de Xynthia ont marqué et marqueront la commune pour de nombreuses années. La question se pose notamment de l'étude de scénarios alternatifs aux « zones noires » qui auraient permis de limiter les déconstructions, réduisant ainsi sensiblement les coûts, et permettant une meilleure utilisation de l'argent public.

L'objectif est donc de quantifier la vulnérabilité des constructions pour leurs occupants afin de comparer l'intérêt de différentes mesures - «zones noires » telles qu'elles ont été appliquées, protection, adaptation, relocalisation, alternatives - que ce soit dans leur efficacité à protéger les populations que dans leurs coûts de mise en œuvre.

Nous nous intéresserons donc à la genèse de la catastrophe, à ses conséquences et à la gestion opérée par l'État afin d'évaluer la pertinence de scénarios alternatifs pour l'adaptation de la commune au risque de submersion marine.

\section{Xynthia À La Faute-sur-Mer : UN ÉVÉNEMENT AUX CONSÉQUENCES MULTIPLES}

\section{Genèse de la catastrophe}

La commune de La Faute-sur-Mer est née en 1953 à la suite de l'indépendance d'un petit hameau dépendant de la commune voisine de La Tranchesur-Mer. Ce hameau était relativement isolé de cette dernière puisque situé sur la flèche sableuse de la pointe d'Arçay. Cette bande de sable de $10 \mathrm{~km}$ de long pour $1 \mathrm{~km}$ de large est orientée nord-sud. Elle s'est créée au cours des trois derniers siècles (Pigeon, 2012) et se présente sous la forme d'un cordon dunaire d'une altitude d'une dizaine de mètres faisant face à l'océan puis d'une zone basse, comprise entre 1 et $5 \mathrm{~m}$ d'altitude le long de l'estuaire du Lay. Les zones les plus basses ont été progressivement déconnectées des incursions récurrentes des eaux de l'estuaire du Lay par une série de digues (Péret et Sauzeau, 2014). Construites par les premiers habitants du hameau de La-Faute-sur-Mer situé à une altitude comprise entre 3 et $5 \mathrm{~m}$, cellesci avaient pour objectif de protéger les troupeaux de bétails (Péret et Sauzeau, 2014).

À la suite de la création de la commune, une expertise menée par des ingénieurs des services de
l'État avait signalé que ces digues n'avaient ni la condition, ni la vocation, de protéger des habitations (Péret et Sauzeau, 2014).

Pourtant, le développement futur de la commune va accorder un rôle de protection majeur à ces digues sans qu'elles fassent pour autant l'objet de travaux de confortement importants.

La commune se présente donc sous la forme d'un territoire de $8,3 \mathrm{~km}^{2}$ dont $80 \%$ se situent à une altitude inférieure à 4,70 m NGF (Creach, 2015), cote retenue pour les Plus Hautes Eaux Connues (PHEC) sur le secteur suite à l'événement Xynthia (DREAL Pays de la Loire, 2014).

«Fille du tourisme» (Cabanne, 1977), La Fautesur-Mer va connaître un développement exponentiel entre 1953 et 2010, développement centré sur l'activité balnéaire. Sa population est ainsi multipliée par trois, tandis que le nombre de constructions résidentielles est multiplié par sept (INSEE, 2014; Vinet et al., 2012). Cela a pour corollaire une très forte représentation des résidences secondaires qui comptent pour $85 \%$ des logements au recensement de 2006 (INSEE, 2014) et un aménagement progressif des zones les plus basses et les plus proches des digues dans une dynamique " [d'urbanisation qui] s'est faite (en partie) en relation inverse du danger » (Vinet et al., 2012). Ainsi, en 2006, la commune comptait 1008 habitants et 3737 logements (INSEE, 2014) dont $89 \%$ étaient susceptibles d'être inondés pour un événement de type Xynthia.

Enfin, au nom d'une forme de tradition architecturale (Péret et Sauzeau, 2014), d'évolutions démographiques (Vinet et al., 2012)1 et des règles d'urbanisme de la commune (Pigeon, 2012), les constructions de plain-pied représentaient $71 \%$ du bâti résidentiel potentiellement exposé au risque de submersion marine en 2006 (Creach, 2015).

\section{Xynthia et ses conséquences directes}

Dans la nuit du 28 février au $1^{\text {er }}$ mars 2010, la tempête Xynthia entraîne la submersion de 41 \% de la commune et de $53 \%$ de ses logements (Devaux et al., 2012). 29 personnes y décèdent, soit $3 \%$ de la population communale. Toutes les victimes ont été piégées à l’intérieur de chez elles (Vinet et al., 2011).

\footnotetext{
1. D'après Vinet et al. (2012) réduction de la taille moyenne du foyer entre 1968 et 2012 et le vieillissement de la population conduit, pour des raisons pratiques, à une préférence pour des constructions de plain-pied.
} 
Si les caractéristiques démographiques de la commune, le moment de survenue de l'inondation et l'absence de consignes claires ont joué un rôle lors de cet événement, les caractéristiques de l'urbanisation de la commune ont été particulièrement mises en avant pour expliquer ce bilan.

Ainsi, l'urbanisation des zones basses, à proximité des digues protégeant la commune de l'estuaire du Lay, et la prédominance des constructions de plainpied ont contribué à former un véritable piège :

- $100 \%$ des victimes sont décédées dans des constructions où la hauteur d'eau a dépassé $1 \mathrm{~m}$ (Vinet et al., 2011);

- $90 \%$ de victimes ont été localisées dans des constructions situées à moins de $400 \mathrm{~m}$ des digues et où l'eau est montée rapidement, surprenant les habitants dans leur sommeil (Vinet et al., 2011);

- $75 \%$ des décès sont intervenus dans des constructions de plain-pied où les personnes n'ont pu que difficilement fuir en hauteur (Vinet et al., 2011).

La localisation et la configuration du bâti résidentiel ont donc créé une situation de danger pour les occupants, seulement compensée par la très forte représentation des résidences secondaires, en partie inoccupées à cette époque de l'année.

\section{Les conséquences indirectes}

Prenant conscience de cette situation, le Président de la République affirme le $1^{\text {er }}$ mars 2010 que « là où il y a risque mortel, personne ne reviendra y habiter; [...] nous n'autoriserons aucune reconstruction en zone mortelle » et de préciser qu' « une cartographie précise est en cours d'élaboration destinée à identifier les sites à fort aléa » (Mercier et Chadenas, 2012).

Cela débouche sur la mise en œuvre des « zones noires », l'État rachetant puis détruisant 552 constructions à La Faute-sur-Mer, soit $15 \%$ des constructions de la commune (Cour des Comptes, 2012; Pitié and Puech, 2010).

Ces déconstructions, en plus de faire disparaître l'ensemble d'un quartier, entraînent une baisse de la population. Ainsi, en 2011, la commune comptait 740 habitants, soit une baisse de $25 \%$ par rapport à 2006 (INSEE, 2014).

Cette baisse n'est pas neutre puisqu'elle fait passer la commune sous la barre de 1000 habitants et, de fait, modifie les règles de scrutins locaux offrant la possibilité de «panacher » les listes présentées ${ }^{2}$. Cela joue lors des élections municipales de 2014 où l'action de l'équipe municipale en place en amont de l'événement Xynthia se voit sanctionnée tandis que le maire sortant - souvent désigné comme l'un des principaux responsables de l'urbanisation des zones inondables - reçoit le soutien d'une partie de ses administrés et est ainsi réélu au conseil municipal dès le premier tour ${ }^{3}$. Cependant, en l'absence de majorité, il n'est pas réélu maire. Il sera ensuite condamné pour homicides involontaires et mise en danger de la vie d'autrui, gardant sa place au conseil municipal mais étant interdit d'exercer une fonction de maire ou d'adjoint ${ }^{4}$.

Ce procès, qui visait également deux adjoints du maire, entrâne une action en justice coûteuse pour les accusés et dont les frais de justice ont été partiellement supportés par la commune ${ }^{5}$.

Par ailleurs, le tribunal administratif de Nantes, dans un jugement confirmé le 6 juillet 2018, a condamné la commune à régler $50 \%$ des 1,86 million d'euros d'indemnités dues aux victimes ${ }^{6}$.

Dans le même temps, la commune voit ses recettes baisser du fait de la disparition des logements détruits dans le cadre de l'action liée aux « zones noires » mais également par la fermeture du camping municipal, totalement inondé lors de Xynthia. Cela a été compensé par une hausse des impôts locaux ${ }^{7}$.

En 2017 a été inauguré un golf dans la zone déconstruite de la commune. Celui-ci permet de donner une nouvelle vocation à cette zone. Par ailleurs, il pourrait permettre une diversification des sources de revenus de la commune ${ }^{8}$.

2. Loi $n^{\circ}$ 2013-403 du 17 mai 2013 relative à l'élection des conseillers départementaux, des conseillers municipaux et des conseillers communautaires, et modifiant le calendrier électoral, 2013.

3. Voir T. Serafini, « À La Faute-sur-Mer, le maire sortant dépassé par ses opposants ", Libération, 28 mars 2014, http://www.liberation.fr/ france/2014/03/28/a-la-faute-sur-mer-le-maire-sortant-depasse-par-sesopposants_991182 (consulté le 31 août 2018).

4. Voir Ouest-France, "Xynthia : René Marratier condamné mais soulagé », 10 avril 2016, [https://www.ouest-france.fr/pays-de-la-loire/vendee/ xynthia-rene-marratier-condamne-mais-soulage-4158328] (consulté le 31 août 2018).

5. Voir le communiqué du maire P. Jouin du 3 octobre 2016 sur le site lafautesurmer.net, http:/www.lafautesurmer.net/2016/10/05/informationde-mairie-de-faute-mer/ (consulté le 31 août 2018).

6. Voir communiqué de presse de la Préfecture de Vendée du 9 juillet 2018 : [http://www.vendee.gouv.fr/IMG/pdf/cp_indemnisation_des_victimes_-_ tempete_xynthia_09-07-2018.pdf] (consulté le 31 août 2018).

7. Voir l'article « À La Faute, le poids de Xynthia sur les finances » sur le site lafautesurmer.net, http://www.lafautesurmer.net/201 1/05/18/3806/ (consulté le 31 août 2018).

8. Voir A. Jarnoux, « La Faute-sur-Mer : sept ans après la tempête Xynthia, un golf est inauguré dans la "cuvette de la mort" », France Bleu Loire 
Enfin, les destructions associées aux «zones noires » ont entraîné une menace de fermeture de l'école ainsi qu'une baisse de fréquentation des commerces ${ }^{9}$.

Ainsi, pour la commune de La Faute-sur-Mer, les conséquences de l'événement Xynthia dépassent largement le bilan humain. Les destructions associées aux «zones noires » marquent et marqueront la commune sur le long terme, comme une cicatrice de l'événement. Il est donc légitime de s’interroger même a posteriori - sur l'intérêt éventuel de mesures alternatives qui auraient permis de limiter ces conséquences à plus long terme tout en répondant au besoin de sécurité évident pour les constructions situées en zone potentiellement inondable.

\section{Mesure de L'impaCt DES « ZONES NOIRES » SUR LA VULNÉRABILITÉ DES CONSTRUCTIONS DE LA COMMUNE}

Si la vulnérabilité d'une partie des constructions résidentielles de La Faute-sur-Mer semble évidente, la question se pose tout de même de la légitimité des déconstructions, au regard notamment des conséquences qu'elles ont entrainées et de l'absence de réflexion sur des mesures alternatives.

Il est donc proposé de comparer l'intérêt de différents scénarios possibles pour réduire la vulnérabilité des constructions pour leurs occupants. Pour cela, il faut disposer d'un outil permettant une telle comparaison. L'indice VIE (Creach et al., 2015) est ainsi présenté. L'état des lieux de la vulnérabilité des constructions telle qu'elle était avant Xynthia et l'impact des « zones noires » sur cette vulnérabilité sont ensuite exposés.

\section{Un outil pour mesurer la vulnérabilité des constructions pour leurs occupants}

Creach et al. (2015) ont proposé une méthodologie permettant d'évaluer la vulnérabilité des constructions résidentielles pour leurs occupants.

Océan, 25 mars 2017, [https://www.francebleu.fr/infos/societe/la-fautesur-mer-sept-ans-apres-la-tempete-xynthia-un-golf-est-inaugure-dans-lacuvette-de-la-mort-1490205635] (consulté le 31 août 2018).

9. Voir A. Négroni, "Cinq ans après Xynthia, La Faute-sur-Mer se meurt », Le Figaro, 27 février 2015, [http://www.lefigaro.fr/actualitefrance/2015/02/27/01016-20150227ARTFIG00331-cinq-ans-apresxynthia-la-faute-sur-mer-se-meurt.php] (consulté le 31 août 2018).
L'intérêt de cette méthode, l'indice VIE (Vulnérabilité intrinsèque extrême), est qu'elle permet de quantifier la vulnérabilité des constructions selon différents scénarios et donc de les comparer entre eux. Elle s'applique à l'échelle de la construction résidentielle ce qui permet de juger de la pertinence de diverses actions de réduction de la vulnérabilité sur chacune des résidences, et d'offrir ainsi un outil d'aide à la décision pour l'adaptation des logements dans un objectif de protection des populations.

Cet indice repose sur quatre critères (figure 1 - planche IV) qui ont été jugés prépondérants dans la construction de la situation de piège lors de l'événement Xynthia (Vinet et al., 2011) :

- La hauteur d'eau potentielle à l'intérieur des constructions : plus cette hauteur d'eau est importante, plus la vulnérabilité des occupants est importante. Au-delà d'un mètre, la vie humaine est en danger (MEDDTL, 2011a).

- La distance des constructions à des digues : plus une construction est proche d'une digue, plus le niveau d'eau est susceptible d'augmenter rapidement à l'intérieur de la construction en cas de défaillance de la digue (rupture ou surverse) et ainsi de créer un effet de surprise pour les occupants.

- Le type architectural des constructions : les constructions pourvues d'un étage ou plus offrent un accès plus aisé pour se réfugier en hauteur que les constructions de plain-pied.

- La proximité des constructions à des zones refuges : plus une construction est proche d'une telle zone plus il sera aisé de s'y réfugier en dernier recours en cas de submersion marine. On entend par zone refuge toute zone située naturellement hors d'eau (altitude supérieure à la cote d'inondation).

Ces critères, appliqués à l'échelle de la construction, s'échelonnent de 0 (absence de vulnérabilité) à 4 (vulnérabilité prononcée) (figure 1 - planche IV), et sont ensuite agrégés selon la formule validée par Creach et al. (2015). Cela permet d'obtenir une note représentant la vulnérabilité des constructions pour leurs occupants, allant de 0 à 12 . Afin d'obtenir une cartographie fine, les constructions résidentielles sont colorées selon quatre classes en fonction de leur note de vulnérabilité :

- Classe verte (indice VIE = 0) : inclut les constructions qui ne sont pas exposées au risque d'inondation par la mer (critère $1=0$ ) et qui ne présentent donc aucune vulnérabilité pour leurs occupants; 
- Classe orange (indice VIE ]0; 5]) : inclut les constructions exposées au risque d'inondation par la mer mais où la vulnérabilité pour les occupants est modérée;

- Classe rouge (indice VIE $[5 ; 8]$ ) : inclut les constructions exposées au risque d'inondation par la mer et où la vulnérabilité est forte mais en principe non létale en l'absence de comportements à risques ou de sur-vulnérabilité (PMR, personnes âgées, enfants);

- Classe noire (indice VIE > 8) : inclut les constructions exposées au risque d'inondation par la mer et où la vulnérabilité des constructions est source de danger mortel pour les occupants. On parlera ici de vulnérabilité « extrême ».

La méthode permet ainsi de dresser des cartes du niveau de vulnérabilité des constructions pour leurs occupants. En plus de pouvoir poser un diagnostic de vulnérabilité des constructions résidentielles, cette méthode permet également de mesurer la vulnérabilité potentielle de ces mêmes constructions pour différents scénarios d'adaptation.

Creach et al. (2016) ont ainsi identifié quatre scénarios permettant de réduire la vulnérabilité des constructions pour leurs occupants. Ces différentes actions permettent d'agir sur l'un des critères de l'indice ce qui offre la possibilité de le recalculer et ainsi de comparer ses résultats avant et après mise en œuvre de l'une des mesures d'adaptation :

- Mesure 1 - rehaussement et confortement des digues : il s'agit d'intervenir sur les ouvrages existants en les rehaussant à la cote du niveau marin centennal intégrant l'élévation du niveau de la mer à l'horizon 2100 et en confortant leur structure. Cette mesure permet d'agir sur le critère 2 de l'indice VIE. Il convient de noter que les digues ne peuvent jamais être considérées comme infaillibles et peuvent même exacerber l'aléa en cas de défaillance (Magnan et Duvat, 2015; Pigeon, 2015; Vinet, 2010). Cette ambivalence de la digue est intégrée dans la mesure puisqu'un ouvrage conforté et réhaussé sera en meilleur état (impact positif sur le critère 2) mais plus haut (impact négatif sur la distance des constructions à la digue).

- Mesure 2 - adaptation architecturale des constructions : il s'agit de construire un étage aux constructions de plain-pied. Cette mesure permet d'agir sur le critère 3 de l'indice VIE.
- Mesure 3 - prévention et évacuation : il s'agit ici de jouer sur deux tableaux. D'une part, elle consiste à renforcer la prévention en établissant un certain nombre de documents réglementaires (PPR-L, Plan de prévention des risques littoraux), d'information (DICRIM, Document d'information communale sur les risques majeurs) et de préparation à la gestion de crise (PCS, Plan communal de sauvegarde) et de mener des exercices d'évacuation pour préparer citoyens, élus et services de secours à l'évacuation. D'autre part, il s'agit de faciliter l'évacuation préventive en proposant des kits de « survie » (tel que proposé par le ministère de l'écologie ${ }^{10}$ ) pour les constructions susceptibles d'être inondées ainsi que l'installation de plateformes refuges permettant une évacuation en dernier recours, de telle sorte que chaque construction soit située à moins de $100 \mathrm{~m}$ d'une zone refuge, naturelle ou artificielle. Cette mesure permet d'agir sur le critère 4 de l'indice VIE. Il convient ici de noter que la commune de La Faute-sur-Mer possédait un PPR-L depuis 2007 (Pigeon, 2012) qui a été révisé en 2011 pour tenir compte de l'événement Xynthia (Chadenas et al., 2013).

- Mesure 4 - relocalisation : il s'agit ici d'envisager la destruction des constructions jugées les plus dangereuses selon le résultat de l'indice VIE (classe noire).

Comme le montre le tableau 1, le coût de mise en œuvre de chacune de ces mesures a été chiffré.

L'ensemble de la méthode permet donc de quantifier la réduction de la vulnérabilité associée à chacun des scénarios d'adaptation au regard du coût nécessaire à la mise en œuvre de ces actions. Il est ainsi possible de comparer leurs intérêts respectifs selon le niveau de protection qu'elles offrent et l'investissement nécessaire pour y parvenir. Il est ainsi possible de porter un jugement a posteriori - ou expost - sur ce qui a été mis en œuvre pour ensuite apporter des pistes de réflexions sur ce qui aurait pu être fait - analyse ex-ante.

\footnotetext{
10. Voir le site gouvernement.fr, «Préparer son kit d'urgence », [https:// www.gouvernement.fr/risques/preparer-son-kit-d-urgence] (consulté le 31 août 2018).
} 


\begin{tabular}{|c|c|c|c|c|}
\hline Mesures et coûts & Description & $\begin{array}{c}\text { Coût } \\
\text { d'investissement }\end{array}$ & $\begin{array}{c}\text { Coût d'entretien } \\
\text { (provision annuelle) }\end{array}$ & Source \\
\hline $\begin{array}{c}\text { Mesure } 1 \\
\text { Protection } \\
\text { Rehaussement \& } \\
\text { confortement des digues }\end{array}$ & $\begin{array}{l}\text { Rehaussement et confortements des ou- } \\
\text { vrages de protection existants (pas de } \\
\text { nouveaux ouvrages). }\end{array}$ & $\begin{array}{l}\text { Digue = } \\
500 € \text { par ml }\end{array}$ & $\begin{array}{c}\text { Digue }=2 \% \\
\text { du coût d'investissement } \\
\text { (10€ par ml/an) }\end{array}$ & $\begin{array}{l}\text { DOLLET \& } \\
\text { SYLLA (2015) }\end{array}$ \\
\hline $\begin{array}{l}\text { Mesure } 2 \\
\text { Accomodation } \\
\text { Adaptation } \\
\text { architecturale }\end{array}$ & $\begin{array}{l}\text { Construction d'une pièce refuge pour les } \\
\text { constructions de plain-pied. Mise en } \\
\text { place d'une fenêtre de toit pour per- } \\
\text { mettre une évacuation verticale. }\end{array}$ & $\begin{array}{c}19265 € \\
\text { par construction }\end{array}$ & Considéré nul & $\begin{array}{c}\text { FESTUOT \& } \\
\text { GUILLAUME (201 }\end{array}$ \\
\hline \multirow{2}{*}{$\begin{array}{l}\text { Mesure } 3 \\
\text { Accomodation } \\
\text { Prévention \& } \\
\text { évacuation }\end{array}$} & \multirow{2}{*}{$\begin{array}{l}\text { Faciliter l'évacuation préventive de la po- } \\
\text { pulation exposée en cas d'alerte en } \\
\text { l'informant (documents réglementaire ), } \\
\text { en la formant (exercice d'évacuation), et } \\
\text { offrir la possibilité de refuge en dernier } \\
\text { recours (plateforme refuge). }\end{array}$} & $\begin{array}{l}\text { Doc. d'information et de } \\
\text { réglementation }=307000 € \\
\text { Exercices d'évac. }=50000 € \\
\end{array}$ & $\begin{array}{l}\text { Prévention = mise à jour des } \\
\text { doc et réalisation d'exercice } \\
\text { d'évac. tous les } 5 \text { ans }=71450 €\end{array}$ & $\begin{array}{l}\text { CDALA } \\
\text { ROCHELLE } \\
\text { (2013) }\end{array}$ \\
\hline & & $\begin{array}{l}\text { Plateforme (unité) }=80000 € \\
\text { Kit d'attente des secours pendant } \\
3 \text { jours (coût par foyer) }=250 €\end{array}$ & $\begin{array}{c}\text { Plateforme }=1 \% \\
\text { du coût d'investissement } \\
(800 € \text { par an) }\end{array}$ & $\begin{array}{l}\text { Entreprise } \\
\text { TDEM }\end{array}$ \\
\hline \multirow{2}{*}{$\begin{array}{c}\text { Mesure } 4 \\
\text { Déplacement } \\
\text { Relocalisation }\end{array}$} & \multirow{2}{*}{$\begin{array}{l}\text { Suppression des constructions les plus } \\
\text { exposées. Nécessite le rachat et la des- } \\
\text { truction de ces habitations. }\end{array}$} & $\begin{array}{l}\text { Rachat = coût au m² } \\
\text { estimé par commune }\end{array}$ & \multirow{2}{*}{ Nul dans le temps } & $\begin{array}{l}\text { Sélection de } \\
\text { sites internet }\end{array}$ \\
\hline & & $\begin{array}{c}\text { Déconstruction }=20000 € \\
\text { par construction }\end{array}$ & & $\begin{array}{l}\text { http://www. } \\
\text { renovation } \\
\text { ettravaux.fr/ }\end{array}$ \\
\hline
\end{tabular}

Tableau 1 : Descriptions et coûts des différentes stratégies d'adaptation pour réduire la vulnérabilité des constructions résidentielles pour leurs occupants (source : auteur, 2017)

Description and costs of different adaptation strategies to reduce building's vulnerability for people (from auteur, 2017)

\section{État des lieux en amont de l'événement Xynthia}

L'application de cette méthode à la situation de La Faute-sur-Mer telle qu'elle était avant la tempête Xynthia montre la très grande vulnérabilité initiale des constructions de la commune (figure 2A - planche V).

Ainsi, ce sont près de la moitié des constructions de la commune qui présentent un niveau de vulnérabilité potentiellement " extrême » $(48 \%)$ et même les trois-quarts si l'on y ajoute les constructions identifiées dans la classe rouge $(81 \%)$. Cette situation s'explique par la topographie basse de la commune, $80 \%$ de la surface de La Faute-sur-Mer étant située sous le niveau marin atteint lors de Xynthia; par la grande proximité de l'urbanisation aux digues protégeant la commune de la rivière du Lay (moins de $20 \mathrm{~m}$ entre les digues et les maisons les plus proches); une majorité de constructions de plain-pied $(71 \%$ des constructions susceptibles d'être inondées); et, corollaire de la topographie de la commune, la faible surface de zones refuges représentées uniquement par le cordon dunaire faisant face à l'océan et par conséquent à l'opposé des constructions les plus proches des digues.

Ainsi, on observe deux zones se distinguant par un niveau de vulnérabilité élevé : la partie sud de la commune (secteur de Claire-Joie) où ont été retrouvées les 29 victimes de la tempête Xynthia; et la partie nord (secteur de la Chenolette). Elles sont toutes deux situées en zones basses et à proximité des digues le long du Lay.

Si le fait que la moitié des constructions de la commune soit identifiée en noir par l'indice VIE peut surprendre et paraître exagéré à l'aune de 29 victimes recensées lors de la tempête Xynthia, ce résultat n'est pas pour autant aberrant.

D'une part, la méthode de l'indice VIE surestime le nombre de constructions susceptibles d'être inondées et les hauteurs d'eau potentielles (critère 1) puisque celles-ci sont calculées en faisant la différence entre la hauteur d'eau théorique et la cote du terrain naturel (Breilh et al., 2013). Il s'agit d'une hypothèse peu réaliste à l'échelle d'un événement de submersion marine mais qui a le mérite de traiter également les constructions selon la diversité des événements possibles (orientation des vents, niveau de surcote, scénarios de défaillance des digues, hauteur d'eau du Lay, etc.). Il s'agit donc du scénario du « pire », dont la réalisation est peu probable lors d'un seul événement d'inondation mais pas impossible pour une succession de submersions.

D'autre part, lors de Xynthia, c'est la partie sud de la commune qui a été la plus sévèrement inondée puisque la submersion marine est arrivée de la mer par l'estuaire du Lay. L'effet de décharge peut être important (Huguet et al., 2017), la submersion de la partie sud ayant conduit à un niveau d'eau du Lay plus faible au nord et donc une submersion moins 
importante. Néanmoins, Pitié et Puech (2010) ont également signalé la zone nord comme étant particulièrement vulnérable du fait de la configuration et de la localisation du bâti. De plus, ces auteurs s'interrogeaient à juste titre sur les conséquences potentielles d'une conjonction entre une submersion marine et une crue du Lay.

Enfin, il convient de noter que $85 \%$ des logements de La Faute-sur-Mer étaient des résidences secondaires lors du recensement de 2006 (INSEE, 2014) et étaient - fort heureusement - en grande partie inoccupées lors du passage de la tempête Xynthia.

Envisager la réduction de la vulnérabilité de la commune était donc essentielle à la suite de l'événement Xynthia afin de limiter les conséquences futures de tels événements. Ceux-ci pourraient augmenter dans un contexte d'élévation du niveau de la mer liée au changement climatique tandis que la population exposée pourrait être plus nombreuse selon la saisonnalité des événements.

\section{Les «zones noires » et leur impact sur la vulnérabilité des constructions}

Faisant face au constat de la dangerosité des constructions pour leurs occupants, l'État décide ainsi de procéder au rachat et à la destruction de 552 constructions dans la cadre de la politique des « zones noires ». Parmi celles-ci, 550 ont pu être caractérisées par l'indice VIE. 414 d'entre-elles étaient identifiées dans la classe noire (75\%) et 136 dans la classe rouge (25\%). La mesure fait ainsi passer la classe noire de 48 à $42 \%$ des constructions de la commune tandis que la classe rouge connaît une hausse de 33 à $35 \%$ (tableau 2 et figure $2 \mathrm{~B}$ - planche $\mathbf{V}$ ).

Par rapport aux résultats de l'indice VIE, la réduction de la vulnérabilité offerte par les « zones noires » est somme toute limitée au regard de la somme investie (160 millions d'euros) du fait de la disparition partielle des constructions présentant le niveau de vulnérabilité le plus élevé.

Précisons que les destructions n'ont pas été la seule réponse apportée suite à l'événement Xynthia. Les digues de la commune ont ainsi été confortées après Xynthia pour un coût de 900000 euros ${ }^{11}$ tan-

11. Voir « 23 millions d'euros investis dans les digues vendéennes depuis Xynthia », Ouest-France, 27 septembre 2013, https://www.ouest-france. fr/pays-de-la-loire/la-roche-sur-yon-85000/23-millions-deuros-investisdans-les-digues-vendeennes-depuis-xynthia-529499 (consulté le 31 août 2018).

\begin{tabular}{|l|c|c|c|c|}
\hline $\begin{array}{l}\text { Indice VIE } \\
\text { (Événement centennal } \\
4,70 \text { m NGF) }\end{array}$ & \multicolumn{2}{|c|}{ État des lieux } & \multicolumn{2}{|c|}{ « Zones noires » } \\
\hline Classe A & 289 & $11 \%$ & 289 & $13 \%$ \\
\hline Classe B & 216 & $8 \%$ & 216 & $10 \%$ \\
\hline Classe C & 885 & $33 \%$ & 749 & $35 \%$ \\
\hline Classe D & 1305 & $48 \%$ & 891 & $42 \%$ \\
\hline $\begin{array}{l}\text { Total des constructions } \\
\text { identifiées }\end{array}$ & 2695 & $100 \%$ & 2145 & $100 \%$ \\
\hline Non identifiées & 1069 & $28 \%$ & 964 & $31 \%$ \\
\hline Total & 3764 & $100 \%$ & 3109 & $100 \%$ \\
\hline $\begin{array}{l}\text { Coût de la mesure } \\
\text { (en million d'€) }\end{array}$ & & 0 & & 160 \\
\hline
\end{tabular}

Tableau 2 : Comparaison des résultats de l'indice VIE entre la situation initiale et la siuation faisant suite à la mise en œuvre des « zones noires » et coût de mise en œuvre (source : auteur, 2015). Comparison of VIE index results between the situation before Storm Xynthia and "black zones" policy (from auteur, 2015).

dis qu'un accompagnement a été mis en place pour les propriétaires souhaitant construire un étage refuge $^{12}$ même si cette action est restée marginale. Enfin un PSR a été adopté en 2011 pour un coût de $157000 €^{13}$. Les bénéfices de ces actions en matière de réduction de la vulnérabilité des constructions pour leurs occupants n'ont pas été quantifiés au travers de l'indice VIE du fait d'un manque d'information à leurs sujets (cote des ouvrages, constructions ayant bénéficié de l'ajout d'un étage).

\section{COMPARAISONS DE SCÉNARIOS ALTERNATIFS}

Cette section propose de comparer l'efficacité de quatre scénarios différents pour réduire la vulnérabilité des constructions pour leurs occupants telle que diagnostiquée en amont de la tempête Xynthia (incluant donc les constructions qui ont par la suite été détruites dans le cadre de la politique des «zones noires »), ainsi que deux scénarios alternatifs. Elle vise à offrir un regard critique sur la mise en œuvre de l'action de l'État dans la gestion post-Xynthia.

12. Voir E. Sérazin, «Xynthia : 8 ans après le drame, seuls $10 \%$ des propriétaires ont réalisé des travaux de sécurité à la Faute-sur-Mer ", France Bleu, 28 février 2018, https://www.francebleu.fr/infos/climat-environnement/xynthia-8-ans-apres-le-drame-seuls-10-des-proprietaires-ont-realise-des-travaux-de-securite-a-la-1519749169 (consulté Le 31 août 2018).

13. Voir S. Amsili et D. Hypolite, "Xynthia : les chiffres de la tempête, un an après », Le Figaro, 22 février 2011, [http://www.lefigaro.fr/ conjoncture/201 1/02/22/04016-20110222ARTFIG00804-xynthia-leschiffres-de-la-tempete-un-an-apres.php] (consulté Le 31 août 2018). 


\section{Rehausser et conforter les digues}

Cette mesure consiste en le rehaussement et le renforcement des digues existantes de la commune. Par rapport à la situation avant la tempête Xynthia, il s'agit de traiter $5,7 \mathrm{~km}$ de digues et 1,4 km de perrés qui étaient en mauvais état et/ou pas à la cote (données issues de SIG Loire). Le coût de ces travaux est estimé à 4 millions d'euros auxquels il est nécessaire de provisionner annuellement $2 \%$ de cette somme pour l'entretien courant (tableau 3).

L'effet de cette mesure est modéré sur les résultats de l'indice VIE. La classe noire passe ainsi de 48 à $45 \%$ tandis que la classe rouge passe de 33 à $38 \%$ (tableau 3 et figure 2C - planche VI). Cela s'explique par l'ambivalence de la mesure : si le confortement et le rehaussement des digues permet de réduire la probabilité d'une défaillance en cas de niveaux d'eau importants, le fait de rehausser l'ouvrage peut avoir un effet pervers en cas de défaillance (par manque d'entretien ou par surverse lié à un événement d'intensité supérieur à celui pour lequel l'ouvrage est dimensionné) puisque la hauteur de chute en arrière de la digue sera plus importante, entraînant un remplissage plus rapide de la zone inondable à même d'augmenter l'effet de surprise.

L'intérêt de cette mesure réside avant tout dans la possibilité de réduire la probabilité des événements de submersion et donc la récurrence des dommages matériels. Néanmoins, dans l'objectif de protéger les populations, ce n'est jamais une mesure suffisante : par définition, les événements entraînant des décès sont des événements "extrêmes " - rares et de fortes intensités - pour lesquels les digues peuvent être source de danger plus que de protection.

\section{Construire un étage aux maisons de plain-pied}

Cette mesure consiste en la création d'un étage refuge aux constructions de plain-pied et susceptibles d'être des pièges pour les occupants (classes noire et rouge).

Le coût d'une telle opération a été chiffré à 20000 euros (Festuot et Guillaume, 2015) par maison à adapter. Cette somme comprend l'ajout d'un étage de $21 \mathrm{~m}^{2}$ sur une construction déjà existante.

\begin{tabular}{|l|c|c|c|c|}
\hline $\begin{array}{l}\text { Indice VIE } \\
\left(\begin{array}{l}\text { Événement centennal } \\
4,70 \mathrm{~m} \text { NGF) }\end{array}\right.\end{array}$ & \multicolumn{2}{|c|}{ État des lieux } & \multicolumn{2}{c|}{$\begin{array}{l}\text { Mesure 1 } \\
\text { Protection }\end{array}$} \\
\hline Classe A & 289 & $11 \%$ & 289 & $11 \%$ \\
\hline Classe B & 216 & $8 \%$ & 172 & $6 \%$ \\
\hline Classe C & 885 & $33 \%$ & 1016 & $38 \%$ \\
\hline Classe D & 1305 & $48 \%$ & 1218 & $45 \%$ \\
\hline $\begin{array}{l}\text { Total des constructions } \\
\text { identifiées }\end{array}$ & 2695 & $100 \%$ & 2695 & $100 \%$ \\
\hline Non identifiées & 1069 & $28 \%$ & 1069 & $28 \%$ \\
\hline Total & 3764 & $100 \%$ & 3764 & $100 \%$ \\
\hline $\begin{array}{l}\text { Coût de la mesure } \\
\text { (en million d'€) }\end{array}$ & & 0 & & 3,9 \\
\hline
\end{tabular}

Tableau 3 : Comparaison des résultats de l'indice VIE entre la situation initiale et la siuation avec mise en œuvre d'un rehaussement et d'un confortement des digues (mesure 1) et coût de mise en œuvre (source : auteur 2015)

Comparison of VIE index results between the situation before Storm Xynthia and the situation with dikes upgrade (from auteur, 2015)

À La Faute-sur-Mer, cela aurait concerné 1637 constructions de plain-pied incluses dans les classes noire et rouge, pour un coût total se montant à 32 millions d'euros (tableau 3).

La mesure offre un gain en matière de réduction de la vulnérabilité des constructions pour leurs occupants puisque la classe noire est amputée de $71 \%$ (tableau 4 et figure 2D - planche VI). Cela se fait principalement au profit de la classe rouge où le niveau de vulnérabilité est moindre.

\begin{tabular}{|l|c|c|c|c|}
\hline $\begin{array}{l}\text { Indice VIE } \\
\text { (Événement centennal } \\
4,70 \text { m NGF) }\end{array}$ & \multicolumn{2}{|c|}{ État des lieux } & \multicolumn{2}{|c|}{$\begin{array}{l}\text { Mesure } 2 \\
\text { Adaptation } \\
\text { architecture) }\end{array}$} \\
\hline Classe A & 289 & $11 \%$ & 289 & $11 \%$ \\
\hline Classe B & 216 & $8 \%$ & 399 & $15 \%$ \\
\hline Classe C & 885 & $33 \%$ & 1626 & $60 \%$ \\
\hline Classe D & 1305 & $48 \%$ & 381 & $14 \%$ \\
\hline $\begin{array}{l}\text { Total des constructions } \\
\text { identifiées }\end{array}$ & 2695 & $100 \%$ & 2695 & $100 \%$ \\
\hline Non identifiées & 1069 & $28 \%$ & 1069 & $28 \%$ \\
\hline Total & 3764 & $100 \%$ & 3764 & $100 \%$ \\
\hline $\begin{array}{l}\text { Coût de la mesure } \\
\text { (en million d'€) }\end{array}$ & \multicolumn{2}{|c|}{31,9} \\
\hline
\end{tabular}

Tableau 4 : Comparaison des résultats de l'indice VIE entre la situation initiale et la siuation avec adaptation architecturale des constructions (mesure 2) et coût de mise en œuvre (source : auteur, 2015).

Comparison of VIE index results between the situation before Storm Xynthia and the situation with building's architectural adaptation (from auteur 2015). 
Cette mesure, en plus de réduire la vulnérabilité des constructions pour leurs occupants, permet d'agrandir les logements par l'ajout d'une pièce. Ainsi, l'investissement consenti offre un intérêt audelà même de la protection face au risque de submersion marine.

\section{Renforcer la prévention et l'évacuation préventive (plateformes refuges)}

Comme mentionné dans la partie «Un outil pour mesurer la vulnérabilité... », cette mesure se décline en deux volets, l'un portant sur une meilleure préparation des acteurs communaux à d'éventuelles submersions, l'autre - plus structurel - portant sur une évacuation facilitée au travers de l'installation de plateformes refuges à intervalle régulier (tous les $200 \mathrm{~m}$ afin qu'une construction soit située à moins de $100 \mathrm{~m}$ d'une zone refuge naturelle ou artificielle). Pour atteindre cet objectif, ce sont 38 plateformes qu'il faudrait édifier sur la commune. Au total, cette mesure aurait un coût total de 4 millions d'euros auxquels il faudrait ajouter une provision annuelle de 10000 euros afin d'entretenir des plateformes et assurer une pérennité des exercices d'évacuation (tableau 5).

Toutefois, la mesure a un impact très modéré sur la vulnérabilité des constructions pour leurs occupants (tableau 5 et figure 2E - planche VII). Cela tient pour partie à l'architecture de l'indice VIE qui minore le poids de la distance à une zone refuge par rapport aux autres critères et au fait que cette mesure n'insiste pas tant sur la réduction de la vulnérabilité des constructions pour leurs occupants que sur l'information et la préparation à l'évacuation. La meilleure façon de prévenir les décès en cas d'inondation est de limiter la présence des populations. Cette mesure est pertinente en appui d'une mesure structurelle (adaptation architecturale, relocalisation ou protection).

\section{Déconstruire les constructions les plus dangereuses}

Cette mesure consiste en la déconstruction des constructions jugées les plus dangereuses, c'est-àdire les constructions identifiées en noir par l'indice VIE. En l'occurrence, il s'agirait de déconstruire les 1305 résidences identifiées comme tel dans la phase de diagnostic soit $48 \%$ des constructions de La Faute-sur-Mer (tableau 6 et figure 2F

\begin{tabular}{|l|c|c|c|c|}
\hline $\begin{array}{l}\text { Indice VIE } \\
\text { (Événement centennal } \\
4,70 \mathrm{~m} \text { NGF) }\end{array}$ & \multicolumn{2}{|c|}{ État des lieux } & \multicolumn{2}{|c|}{$\begin{array}{c}\text { Mesure 3 } \\
\text { Adaptation } \\
\text { prév. et évacua- } \\
\text { tion) }\end{array}$} \\
\hline Classe A & 289 & $11 \%$ & 289 & $11 \%$ \\
\hline Classe B & 216 & $8 \%$ & 230 & $9 \%$ \\
\hline Classe C & 885 & $33 \%$ & 920 & $34 \%$ \\
\hline Classe D & 1305 & $48 \%$ & 1256 & $47 \%$ \\
\hline $\begin{array}{l}\text { Total des constructions } \\
\text { identifiées }\end{array}$ & 2695 & $100 \%$ & 2695 & $100 \%$ \\
\hline Non identifiées & 1069 & $28 \%$ & 1069 & $28 \%$ \\
\hline Total & 3764 & $100 \%$ & 3764 & $100 \%$ \\
\hline $\begin{array}{l}\text { Coût de la mesure (en } \\
\text { million d'€) }\end{array}$ & 0 & \multicolumn{2}{|c|}{3,9} \\
\hline
\end{tabular}

Tableau 5 : Comparaison des résultats de l'indice VIE entre la situation initiale et la siuation avec renforcement de la prévention et de l'évacuation préventive (mesure 3) et coût de mise en œuvre (source : auteur, 2015).

Comparison of VIE index results between the situation before Storm Xynthia and the situation with improvement of prevention and warning (from auteur, 2015).

- planche VII). On peut évidemment être critique quant à la pertinence de cette mesure, notamment d'un point de vue social ou économique pour la vie de la commune, eu égard aux conséquences des «zones noires».

Pour un coût total estimé à 320 millions d'euros c'est évidemment la mesure la plus efficace -puisque la plus radicale - avec la disparition de la totalité des constructions les plus dangereuses (tableau 5).

\begin{tabular}{|l|c|c|c|c|}
\hline $\begin{array}{l}\text { Indice VIE } \\
\text { (Événement centennal } \\
4,70 \text { m NGF) }\end{array}$ & \multicolumn{2}{|c|}{ État des lieux } & \multicolumn{2}{|c|}{$\begin{array}{l}\text { Mesure 4 } \\
\text { Déplacement }\end{array}$} \\
\hline Classe A & 289 & $11 \%$ & 289 & $21 \%$ \\
\hline Classe B & 216 & $8 \%$ & 216 & $16 \%$ \\
\hline Classe C & 885 & $33 \%$ & 885 & $64 \%$ \\
\hline Classe D & 1305 & $48 \%$ & 0 & $0 \%$ \\
\hline $\begin{array}{l}\text { Total des constructions } \\
\text { identifiées }\end{array}$ & 2695 & $100 \%$ & 1390 & $100 \%$ \\
\hline Non identifiées & 1069 & $28 \%$ & 1069 & $43 \%$ \\
\hline Total & 3764 & $100 \%$ & 2459 & $100 \%$ \\
\hline $\begin{array}{l}\text { Coût de la mesure } \\
\text { (en million d'€) }\end{array}$ & & 0 & 320,5 \\
\hline
\end{tabular}

Tableau 6 : Comparaison des résultats de l'indice VIE entre la situation initiale et la siuation déconstruction des bâtiments les plus dangereux (mesure 4) et coût de mise en œuvre (source : auteur, 2015).

Comparison of VIE index results between the situation before Storm Xynthia and the situation relocation of the most dangerous buildings (from auteur, 2015). 


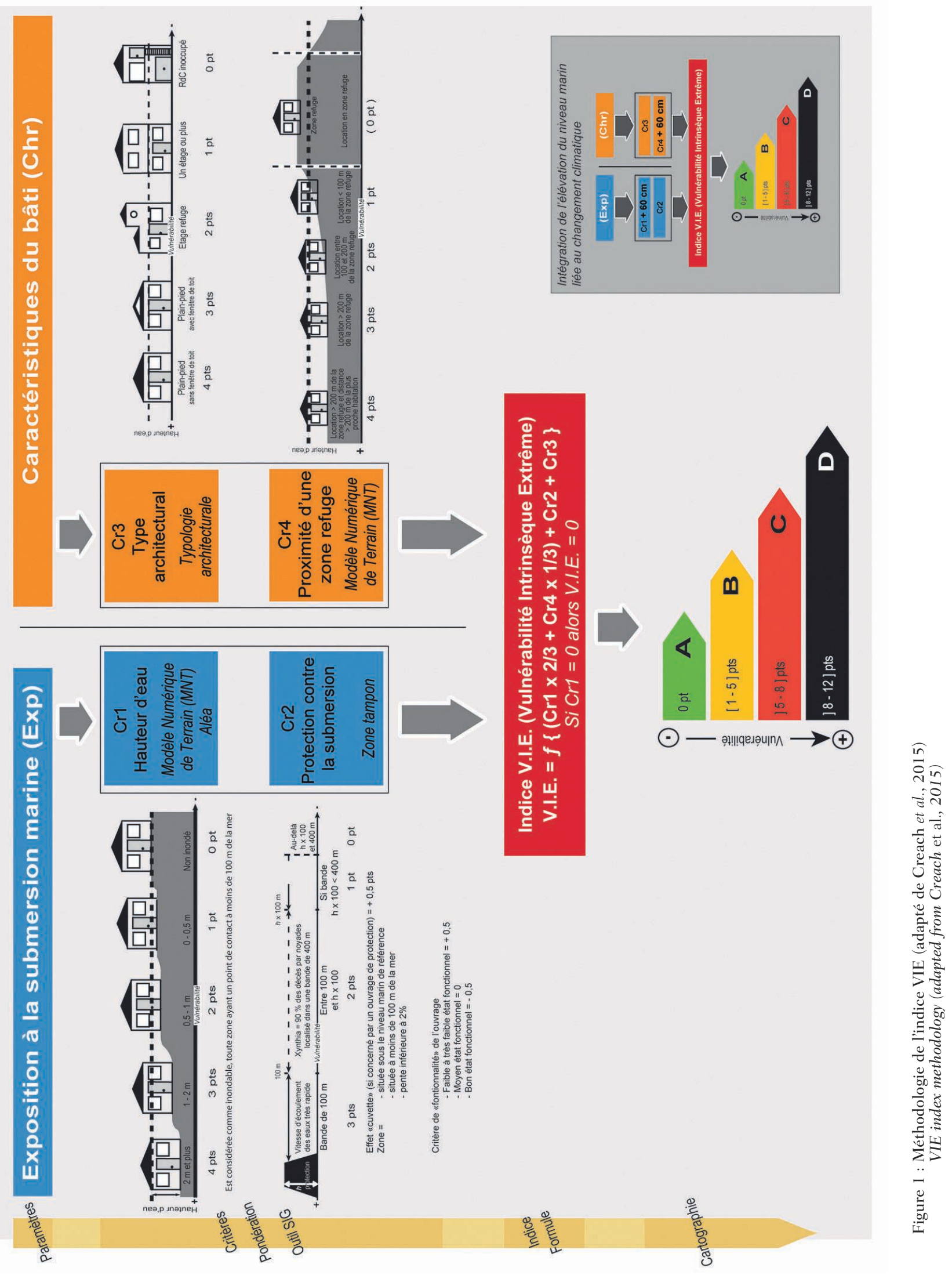



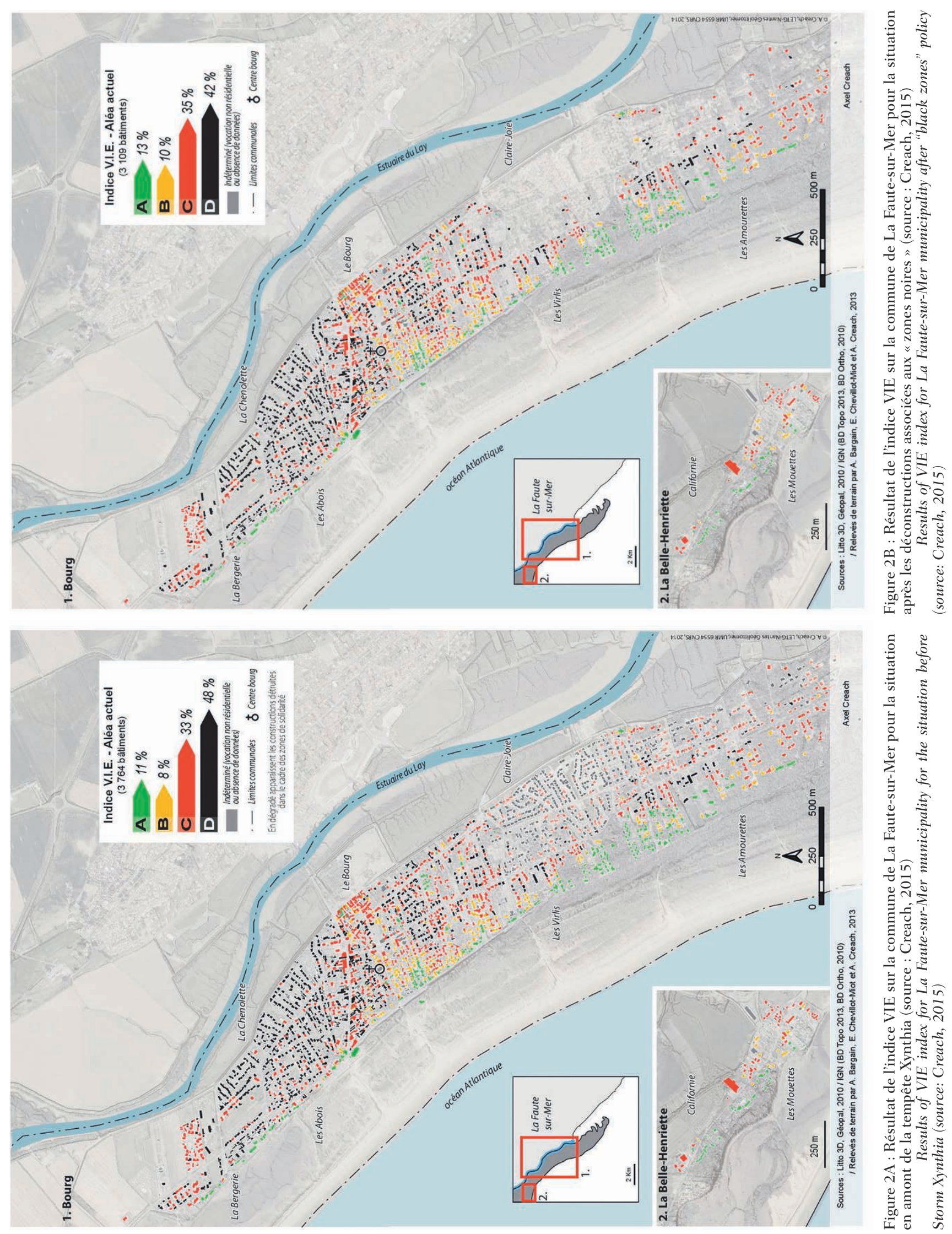

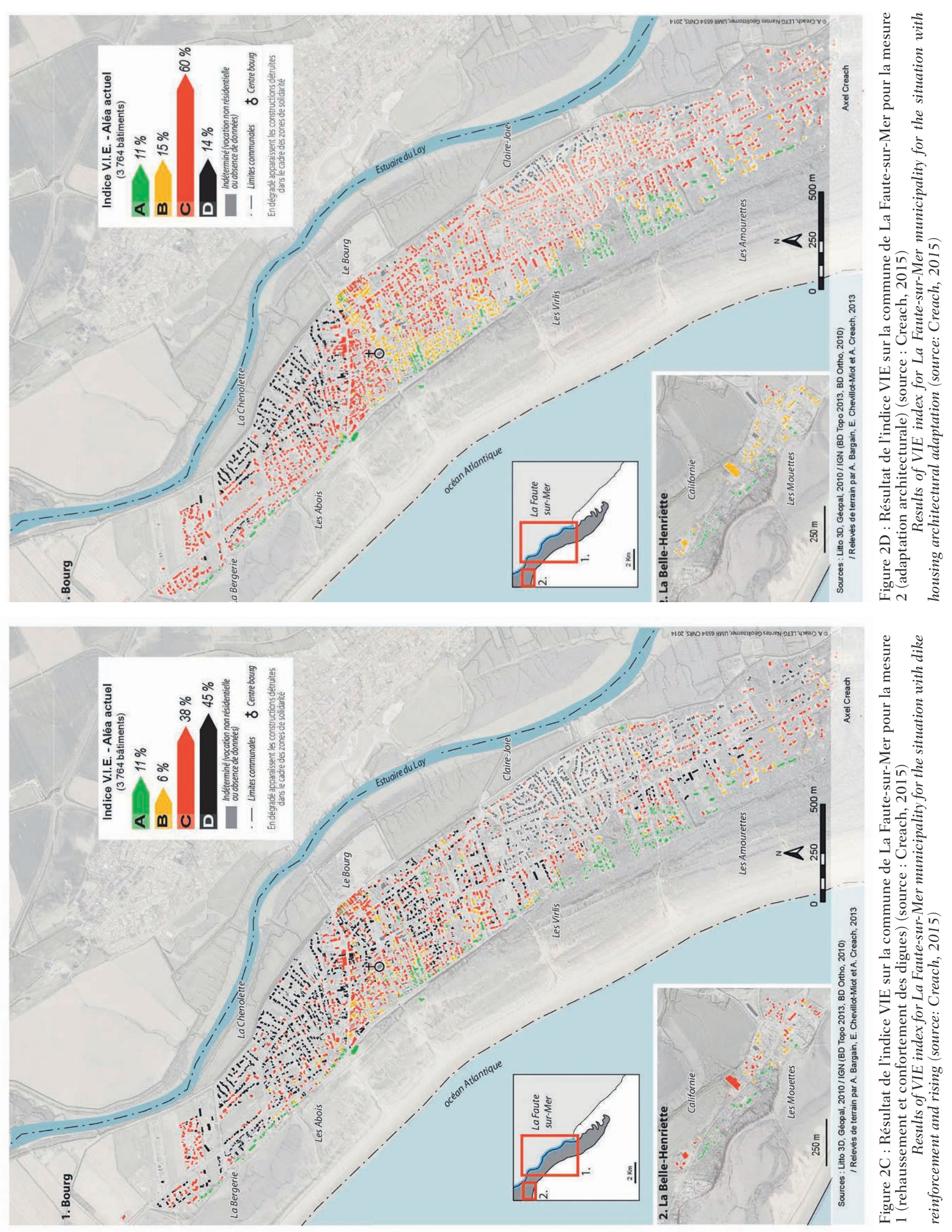

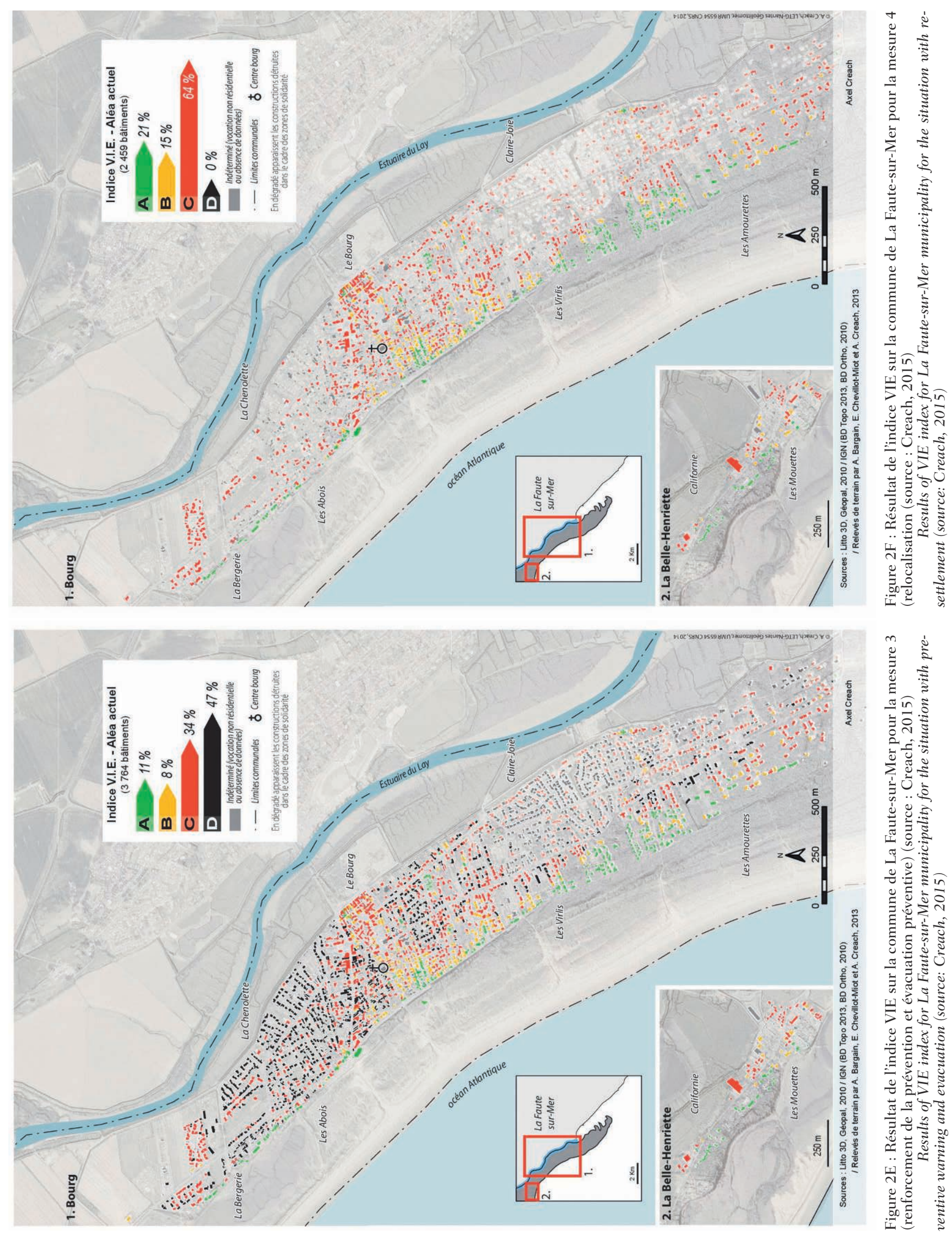

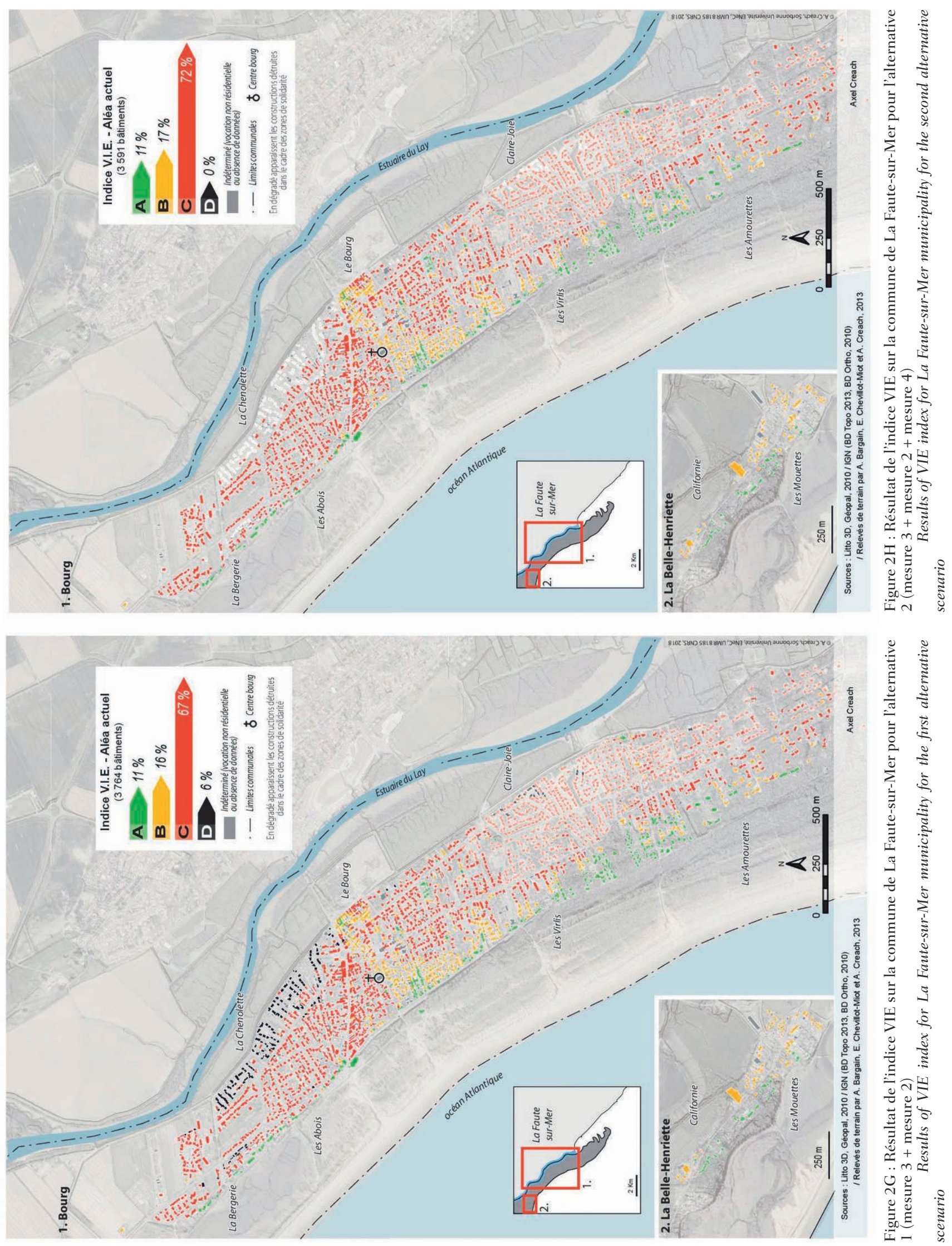



\section{Associer les mesures pour en augmenter l'efficacité : deux scénarios alternatifs}

Dans les faits, la meilleure manière de protéger les populations est d'associer différentes mesures (Klein et al., 2001).

Associer différentes mesures offre potentiellement un double intérêt : augmenter l'efficacité des mesures dans l'objectif de protéger les populations tout en diminuant le coût de mise en œuvre de ces actions.

Deux alternatives sont ici proposées :

-Alternative 1 : associer prévention et évacuation préventive (mesure 3) et adaptation architecturale des constructions (mesure 2). Il s'agit donc de renforcer la préparation à l'inondation tout en offrant la possibilité au maximum de se réfugier, que ce soit chez soi (construction d'un étage refuge) ou à proximité (plateforme refuge).

- Alternative 2 : associer prévention et évacuation préventive (mesure 3 ), adaptation architecturale des constructions (mesure 2) et déconstruction des biens les plus dangereux. Dans l'esprit de l'alternative ci-dessus, il s'agit de limiter au maximum l'exposition à un risque potentiellement mortel en déconstruisant les constructions toujours identifiées dans la classe noire de l'indice VIE malgré un renforcement de la prévention et l'adaptation architecturale des biens.

La première alternative offre une nette réduction du nombre de constructions les plus dangereuses par rapport à la situation sans mesure avant Xynthia, la classe noire passant de 1305 à 173 constructions (tableau 7 et figure $2 \mathrm{G}$ - planche VIII). Il est intéressant de noter que ce résultat est proche de l'adaptation architecturale seule (mesure 2) mais que l'alternative proposée offre un résultat optimisé : pour un surcoût de $12 \%$ par rapport à l'adaptation architecturale seule (32 millions d'euros pour l'adaptation architecturale, 35,7 millions d'euros pour l'alternative proposée) elle offre une réduction du nombre de constructions les plus dangereuses (classe noire) $16 \%$ meilleure $(71 \%$ de réduction pour l'adaptation architecturale, $87 \%$ pour l'alternative).

L'alternative 2 cherche, elle, à atteindre un résultat similaire à la relocalisation (suppression des constructions les plus dangereuses) tout en maintenant un maximum de logements grâce à une adaptation préalable. Ce scénario permet ainsi de supprimer toutes les constructions de la classe noire (tableau 7 et figure $2 \mathrm{H}$ - planche VIII) en procédant à la déconstruction de 173 constructions (contre 1305 pour la mesure de relocalisation telle que proposée dans la partie «Déconstruire les constructions les plus dangereuses ». L'objectif est donc atteint pour un coût quatre fois inférieur à la relocalisation seule (l'alternative 2 revient à un coût total de 76,4 millions d'euros contre 320,5 millions d'euros pour la relocalisation) tout en permettant un maintien des habitations et de leurs occupants. Ce coût est également plus de deux fois inférieur à celui des « zones noires » (160 millions d'euros).

\begin{tabular}{|c|c|c|c|c|c|c|}
\hline \multirow{2}{*}{$\begin{array}{l}\text { Indice VIE } \\
\text { (Événement } \\
\text { centennal } \\
4,70 \text { m NGF) } \\
\text { Classe A }\end{array}$} & \multicolumn{2}{|c|}{ État des lieux } & \multicolumn{2}{|c|}{ Alternative 1} & \multicolumn{2}{|c|}{ Alternative 2} \\
\hline & 289 & $11 \%$ & 289 & $11 \%$ & 289 & $11 \%$ \\
\hline Classe B & 216 & $8 \%$ & 422 & $16 \%$ & 422 & $17 \%$ \\
\hline Classe C & 885 & $33 \%$ & 1811 & $67 \%$ & 1811 & $72 \%$ \\
\hline Classe D & 1305 & $48 \%$ & 173 & $6 \%$ & 0 & $0 \%$ \\
\hline $\begin{array}{l}\text { Total des } \\
\text { constructions } \\
\text { identifiées }\end{array}$ & 2695 & $100 \%$ & 2695 & $100 \%$ & 2522 & $100 \%$ \\
\hline Non identifiées & 1069 & $28 \%$ & 1069 & $28 \%$ & 1069 & $30 \%$ \\
\hline Total & 3764 & $100 \%$ & 3764 & $100 \%$ & 3591 & $100 \%$ \\
\hline $\begin{array}{l}\text { Coût de la } \\
\text { mesure } \\
\text { (en million d'€) }\end{array}$ & \multicolumn{2}{|c|}{0} & \multicolumn{2}{|c|}{35,7} & \multicolumn{2}{|c|}{76,4} \\
\hline
\end{tabular}

Tableau 7 : Comparaison des résultats de l'indice VIE entre la situation initiale et les deux scénarios alternatifs et coûts de mise en œuvre source : (auteur 2015).

Comparison of VIE index results between the situation before Storm Xynthia and the situation for two alternative scenarios (from auteur 2015).

\section{RÉFLEXIONS SUR L'ADAPTATION DES CONSTRUCTIONS}

Ainsi il apparaît que, en plus des « zones noires », différents scénarios pour réduire la vulnérabilité des constructions pour leurs occupants sont envisageables ainsi qu'une combinaison de mesures entre elles. Ceux-ci offrent une réduction de la vulnérabilité différente (réduction de la classe noire de $4 \%$ à $100 \%$ selon les scénarios) ainsi que des coûts de mise en ouvre allant de 4 à 320 millions d'€. Il s'agit donc de donner quelques clés pour comparer l'intérêt de ces divers scénarios de réduction de la vulnérabilité. 
Creach et al. (en cours de publication, sous presse) ont proposé une première approche afin de mettre en regard l'efficacité des scénarios à réduire la vulnérabilité des constructions pour leurs occupants et leurs coûts de mise en œuvre. L'efficacité des scénarios est évaluée au travers de la réduction de constructions dangereuses obtenue par rapport à la situation sans mesure (état des lieux). Cette mesure de l'efficacité est obtenue grâce à la formule suivante :

$$
\Delta \mathrm{R}=\frac{n b_{\text {avec adap }}-n b_{\text {sans adap }}}{n b_{\text {avec adap }}} \times 100
$$

où $n b$ avec adap est le nombre de constructions identifié dans la classe noire après mise en œuvre d'une mesure d'adaptation et $n b$ sans adap est le nombre de constructions identifié dans la classe noire dans la situation sans mesure (état de lieux) ce qui permet d'obtenir un taux de réduction des constructions les plus dangereuses exprimé en pourcentage.

La figure 3 permet de projeter les différents scénarios selon leurs coûts (axe des ordonnées, échelle semi-logarithmique) et leurs efficacités exprimées en pourcentage de réduction du nombre de constructions les plus dangereuses. Ainsi, plus un scénario sera positionné dans la partie supérieure gauche du graphique, plus il sera onéreux dans sa mise en œuvre pour une efficacité limitée; à l'inverse, plus un scénario sera positionné dans la partie inférieure droite, plus il aura une efficacité élevée pour un coût de mise en œuvre limitée.

Les mesures « structurelles » (celles portant directement sur les constructions : adaptation architecturale et relocalisation) apparaissent globalement efficaces mais onéreuses : la relocalisation apparaît comme la solution de la sécurité à « tout prix » avec une disparition totale des constructions les plus dangereuses mais pour un investissement élevé (320 millions d'euros). De plus, cela entraînerait la disparition de près de la moitié des constructions de la commune, ce qui apparaît peu souhaitable d'un point de vue économique et social. L'adaptation architecturale offre ainsi un compromis avec une réduction de $71 \%$ des constructions les plus dangereuses pour un investissement dix fois inférieur (32 millions d'euros).

Les scénarios de protection et de prévention présentent un coût bien plus limité (3,9 millions d'euros) mais pour une efficacité relative (respectivement 7 et $4 \%$ ). Pour ce qui concerne la protection, ce résultat s'explique par le fait que, malgré la réduction d'une probabilité de défaillance de l'ouvrage rehaussé et conforté, la digue ne peut jamais être considérée comme un élément de protection suffisant lorsqu'il s'agit d'enjeux humains. Celle-ci offre un gain substantiel pour protéger les biens face à des événements fréquents et modérés. La mesure de prévention porte, quant à elle, avant tout sur une meilleure préparation des acteurs concernés en cas

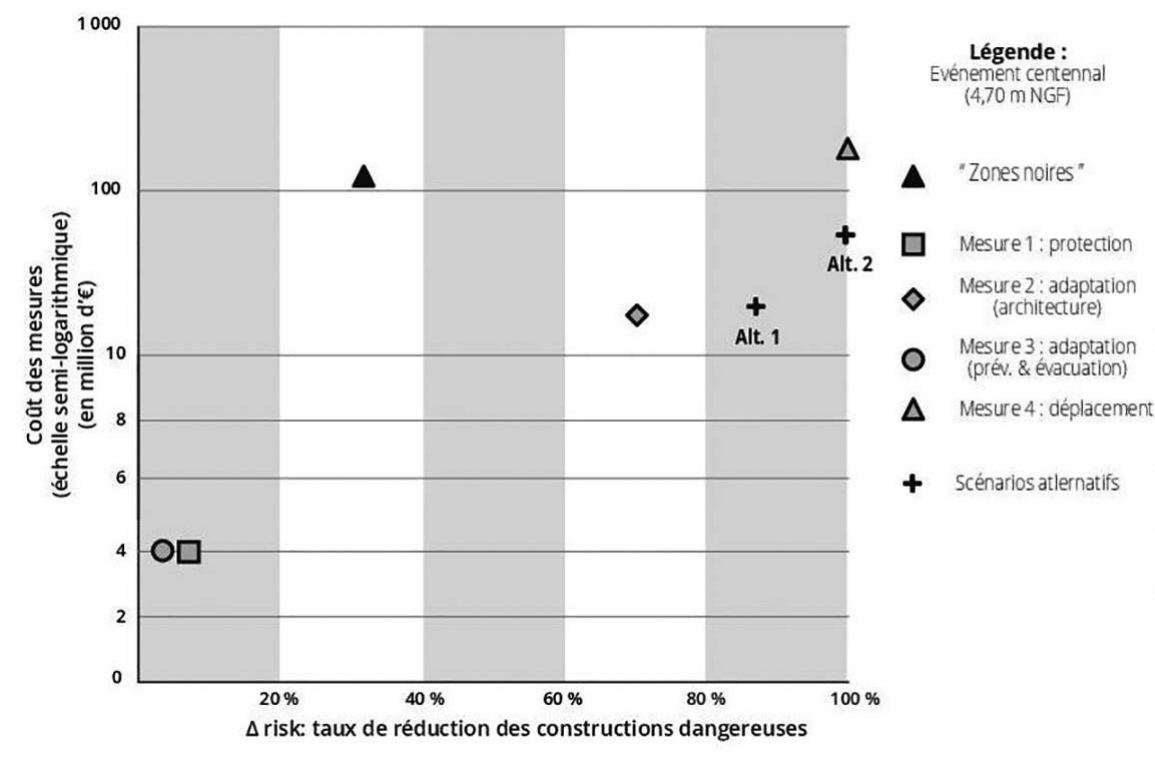

Figure 3 : Comparaison des différentes mesures suivant leurs coûts et leurs efficacités à réduire la vulnérabilité des constructions pour leurs occupants

Comparison of different strategies depending on their costs and efficiency to reduce building's vulnerability for people 
de submersion marine et non pas directement sur une modification des constructions résidentielles. Ceci explique son efficacité limitée. Néanmoins, son coût modeste et la conscience du risque qu'elle est susceptible d'offrir en font une mesure à envisager de manière systématique.

La meilleure façon de réduire la vulnérabilité des constructions résidentielles pour leurs occupants tout en limitant les coûts de mise en œuvre consiste en un mariage de ces différentes mesures. Ainsi, deux scénarios alternatifs ont été proposés, tous deux basés sur un renforcement de la prévention - dont le coût est limité et le bénéfice potentiel élevé - et l'adaptation architecturale qui offre un compromis à la relocalisation. La seconde alternative prévoit, en complément, la destruction des bâtiments dont le niveau de vulnérabilité serait toujours trop élevé. Ces deux scénarios permettent d'atteindre des niveaux d'efficacité supérieurs à $85 \%$ (alternative 1) pour des coûts compris entre 36 (alternative 1) et 76 millions d'euros (alternative 2, pour laquelle le niveau d'efficacité est de $100 \%$ ). La seconde alternative offre ainsi un niveau de protection optimal en supprimant intégralement la classe des constructions les plus dangereuses, tout en limitant le nombre de déconstructions, ce qui permet un maintien de la population de la commune.

La mesure de protection n'est pas intégrée dans ces scénarios alternatifs car elle ne répond pas directement à un objectif de protection des populations mais avant tout à la protection des biens. De plus, on peut considérer que cette mesure est désormais effective suite aux travaux menés après l'événement Xynthia sur la digue est de la commune.

\section{Conclusion}

La commune de La Faute-sur-Mer a été doublement touchée par la tempête Xynthia. Elle a d'abord connu un lourd bilan humain : 29 décès pour une population de 1008 habitants. Elle a ensuite été particulièrement concernée par les «zones noires » mises en place par l'État pour supprimer la dangerosité des constructions pour leurs occupants : $15 \%$ de son parc résidentiel a été détruit dans ce cadre. Ces déconstructions ont entrainé des conséquences secondaires telles qu'une baisse de la population et des revenus fiscaux de la commune, un impact sur l'activité économique et ont pu être, à bien des égards, perçues comme un second traumatisme à la suite de la tempête.

Si les déconstructions telles que proposées par l'État sont bien l'une des réponses possibles pour limiter la vulnérabilité des constructions pour leurs occupants, force est de constater que ces «zones noires » n'ont pas complètement atteint leur objectif : au-delà des débats sur le bien-fondé d'une telle mesure, nous avons pu remarquer que la disparition de 552 constructions pour un coût total de 160 millions d'euros n'avait réduit que partiellement le nombre de constructions pouvant potentiellement exposer leurs occupants à un risque mortel en cas d'inondation (réduction de $32 \%$ ).

L'intérêt était donc de comparer la pertinence d'autres solutions possibles permettant de répondre au besoin de réduire la vulnérabilité des constructions pour leurs occupants.

Ainsi, ont été comparées quatre mesures différentes et deux scénarios alternatifs proposant une association de ces mesures. Le rehaussement et le renforcement des digues n'apparaissent pas comme une mesure suffisante dans l'unique but de protéger les populations tandis que l'amélioration de la prévention et de l'évacuation préventive n'apporte pas directement un gain de réduction de la vulnérabilité des constructions pour leurs occupants. Toutefois, le faible coût de cette seconde mesure en fait une action essentielle en complément d'actions plus structurelles. La déconstruction apparaît comme une mesure radicale, permettant une efficacité maximale en supprimant totalement les constructions les plus dangereuses, mais pour un coût élevé et un impact conséquent sur la commune. L'adaptation architecturale présente une forme de compromis, permettant le maintien des constructions et un coût bien plus modeste mais ne supprime pas totalement la dangerosité des constructions.

La mise en œuvre d'alternatives agrégeant ces différentes mesures offre une vraie plus-value permettant tout à la fois d'augmenter l'efficacité de la réduction de vulnérabilité pour un coût inférieur tout en maintenant un maximum de constructions. Ainsi, associer renforcement de la prévention, adaptation architecturale et déconstruction permet de supprimer totalement les constructions les plus dangereuses (tout comme la mesure de déconstruction appliquée seule) mais pour un coût quatre fois inférieur (76,4 millions d'euros contre 
320,5 millions d'euros pour la déconstruction seule) tout en limitant les déconstructions à 173 habitations (contre 1305 pour la déconstruction seule). C'est également deux fois moins onéreux que le coût des «zones noires » alors que l'efficacité est bien meilleure $(0 \%$ de constructions dangereuses contre $42 \%$ pour les «zones noires ») et un nombre de déconstructions bien inférieur ( 173 contre 552 pour les « zones noires »).

Si ces conclusions ne modifieront en rien ce qui a été fait à La Faute-sur-Mer à la suite de la tempête Xynthia, elles présentent tout de même deux intérêts majeurs.

Tout d'abord elles éclairent sur le risque de «maladaptation » qui peut être défini comme une «utilisation inefficace de ressources comparativement à d'autres options d'utilisation» (MEDDTL, 2011b). Le terme est peut-être fort pour ce qui concerne les « zones noires » puisqu'elles atteignent partiellement leur objectif, mais au prix d'une somme supérieure à celles d'alternatives qui auraient pu s'avérer aussi, voire plus, efficaces. Cette situation peut être mise sur le compte d'une "précipitation qu'on peut estimer excessive »(Cour des Comptes, 2012) de l'État dans la mise en œuvre des "zones noires » pour lesquelles l'intérêt d'alternatives n'a pas été étudié. Ainsi, le risque de «mal-adaptation » est plus élevé lorsque cette adaptation est dite « réactive ", ou "subie », faisant suite à un événement marquant, ce qui est le cas avec les « zones noires » qui relèvent d'une réaction « à chaud ».

Lélévation du niveau de la mer liée au changement climatique et l'importance des constructions résidentielles exposées au risque de submersion marine sur la côte atlantique française (136711 bâtiments [CETMEF et al., 2009], parmi lesquelles $22 \%$ sont de type plain-pied [MEDDE, 2012]) augmentent la probabilité d'événements similaires à Xynthia. L'enjeu est bien d'anticiper l'adaptation afin de se prémunir des conséquences futures de tels événements. Il s'agit donc d'adopter une démarche d'adaptation "préventive» (Nicholls, 2011) pour laquelle les conclusions de ce travail peuvent être utiles afin de proposer des solutions efficaces pour une utilisation optimale de l'argent public tout en maintenant au maximum les populations sur les territoires exposés.

\section{Bibliographie}

Boudou M., 2015. Approche multidisciplinaire pour la caractérisation d'inondations remarquables - Enseignements tirés de neuf évènements en France (1910-2010). Thèse de doctorat en géographie, Université Paul Valéry-Montpellier III, 463 p.

Breilh J.-F., Chaumillon E., Bertin X., Gravelle M., 2013. Assessment of static flood modeling techniques: application to contrasting marshes flooded during Xynthia (western France), Natural Hazards and Earth System Sciences, $\mathrm{n}^{\circ}$ 13, vol. 6, p. 1595-1612. DOI : 10.5194/nhess-13-1595-2013

Burby R.J., 2006. Hurricane Katrina and the paradoxes of government disaster policy: bringing about wise governmental decisions for hazardous areas, Annals of the American Academy of Political and Social Science, $\mathrm{n}^{\circ} 604$, vol. 1, p. 171-191. DOI : 10.1177/0002716205284676

Cabanne C., 1977. L'évolution récente de l'Aiguillon-La Fautesur-Mer (Vendée), Norois, no 95, vol. 3, p. 37-55. DOI : 10.3406/noroi.1977.3634

CETMEF, CETE Méditerranée, CETE Ouest, 2009. Vulnérabilité du territoire National aux risques littoranx, CETMEF/ DLCE, 163 p.

Chadenas C., Creach A., Mercier D., 2013. The impact of storm Xynthia in 2010 on coastal flood prevention policy in France, Journal of Coastal Conservation, $\mathrm{n}^{\circ}$ 8, vol. 18, p. 529-538. DOI : 10.1007/s1 1852-013-0299-3.

Chauveau E, Chadenas C., Comentale B., Pottier P., Blanleil A., Feuillet T., Mercier D., Pourinet L., Rollo N., Tillier I., 2011. Xynthia: leçons d'une catastrophe, CyberGeo: European Journal of Geography, p. 1-25. DOI : 10.4000/cybergeo.23763.

Chauvet A., Renard J., 1978. La Vendée. Le Pays. Les hommes, Éditions du Cercle d'or, Les Sables-d'Olonne, 181 p.

Cour des Comptes, 2012. Les enseignements des inondations de 2010 sur le littoral atlantique (Xynthia) et dans le Var, Rapport public thématique, Cours des Comptes, 299 p.

Creach A., 2017. Vulnérabilité du bâti résidentiel pour leurs occupants : diagnostic et adaptation, Fiche outil $n^{\circ} 3, \mathrm{~A}$ MOving COast in the MEDiterranean (MOCOMED), 5 p.

Creach A., 2015. Cartographie et analyse économique de la vulnérabilité du littoral atlantique français face au risque de submersion marine. Thèse de doctorat en géographie Université de Nantes, 570 p. (2 volumes)

Creach A, Bastidas-Arteaga E., Pardo S., Mercier D., 2016. Comparaison du coût de différentes mesures de protection de la vie humaine face au risque de submersion marine, Paralia, $n^{\circ}$ 14, p. 631-640. DOI : 10.5150/jngcgc.2016.070

Creach A., Bastidas-Arteaga E., Pardo S., Mercier D., (sous presse). Vulnerability and costs of adaptation strategies for housing subjected to flood risks: application to La Guérinière France, Marine Policy, https://doi.org/10.1016/j.marpol.2019.02.010.

Creach A., Pardo S., Gulllotreau P., Mercier D., 2015. The use of a micro-scale index to identify potential death risk areas due to coastal flood surges: lessons from Storm Xynthia on the French Atlantic coast, Natural Hazards, vol 77, $\mathrm{n}^{\circ} 3$, p. 1679-1710. DOI : 10.1007/s 1 1069-015-1669-y0 
Devaux E., Désiré G., Boura C., Lowenbruck J., Bérenger N., Rouxel N., Romain N., 2012. La tempête Xynthia du 28 février 2010 - Retour d'expérience en Loire-Atlantique et Vendée - Volet hydaulique et ouvrages de protection, Retour d'expérience, CETE-Ouest / DREAL Pays-de-la-Loire / DDTM Loire-Atlantique / DDTM Vendée, 78 p.

DREAL Pays DE La LoIRe, 2014. Rapport de présentation de la cartographie du risque de submersion marine sur le secteur de la baie de L'Aiguillon, Rapport final pour la Directive inondations Bassins Loire-Bretagne, DREAL Pays de la Loire, 106 p.

Festuot R., Guillaume L., 2015. Etude technico économique sur la réduction de la vulnérabilité des constructions de plain pied soumise à des risques de submersions. Mémoire de Master, Université de Nantes, 56 p.

Feuillet T., Chauveau É., Pourinet L., 2012. Xynthia est-elle exceptionnelle? Réflexions sur l'évolution et les temps de retour des tempêtes, des marées de tempête, et des risques de surcotes associés sur la façade atlantique française. Norois, n 222, p. 27-44. DOI : 10.4000/norois.3866

Funabashi H., 2012. Why the Fukushima nuclear disaster is a man-made calamity, International Journal of Japanese Sociology, no 21, vol. 1, p. 65-75. DOI : $10.1111 /$ j.14756781.2012.01161.x

Huguet J.-R., Bertin X., Arnaud G., 2017. Managed realignment to mitigate storm-induced flooding: A case study in La Faute-sur-mer, France, Coastal Engineering, vol. 134, p. 168-176. DOI : 10.1016/j.coastaleng.2017.08.010

INSEE, 2014. Recensement général de la population 2011, Publié le 26 juin 2014, en ligne.

Jonkman S.N., Kelman I., 2005. An analysis of the causes and circumstances of flood disaster deaths, Disasters, $n^{\circ} 29$, vol. 1, p. 75-97. DOI : 10.1111/j.0361-3666.2005.00275.x

Klein R.J.T., Nicholls R.J., Ragoonaden S., Capobianco M., Aston J., Buckley E.N., 2001. Technological Options for Adaptation to Climate Change in Coastal Zones, Journal of Coastal Research, vol. 17, n³, p. 531-543.

Kolen B., Slomp R., Jonkman S.N., 2013. The impacts of storm Xynthia February 27-28, 2010 in France: lessons for flood risk management, Journal of Flood Risk Management, vol. 6, nº 3, p. 261-278. DOI : 10.1111/jfr3.12011

Magnan A., Duvat V., 2015. La fabrique des catastrophes « naturelles », Natures Sciences Sociétés, vol. 23, n² p. 97-108. DOI 10.1051/nss/2015033.

MEDDE, 2012. Mieux savoir pour mieux agir : Principaux enseignements de la première évaluation des risques d'inondation sur le territoire français - EPRI 2011, Rapport n 12009, MEDDE, $72 \mathrm{p}$.
MEDDTL, $2011 \mathrm{a}$. Circulaire du 27 juillet 2011 relative à la prise en compte du risque de submersion marine dans les plans de prévention des risques naturels littoraux, NOR : DEVP1119962C, MEDDTL, 19 p.

MEDDTL, $201 \mathrm{lb}$. Plan national d'adaptation de la France aux effets du changement climatique - 2011-2015, MEDDTL, $188 \mathrm{p}$.

Mercier D., Chadenas C., 2012. La tempête Xynthia et la cartographie des «zones noires » sur le littoral français : analyse critique à partir de l'exemple de La Faute-sur-Mer (Vendée), Norois, no 222, p. 45-60. DOI : 10.4000/norois.3895

Nicholls R., 2011. Planning for the Impacts of Sea Level Rise, Oceanography, vol. 24, nº 2, p. 144-157. DOI : 10.5670/ oceanog.2011.34

Péret J., Sauzeau T., 2014. Xynthia ou la mémoire réveillée. Des villages charentais et vendéens face à l'océan (XVIII ${ }^{e}$ XXI ${ }^{e}$ siècles). Éditions Geste, La Crèche, 296 p.

Pigeon P., 2012. Apports de la résilience à la géographie des risques : l'exemple de La Faute-sur-Mer (Vendée, France), VertigO - la revue électronique en sciences de l'environnement, vol. 12, $\mathrm{n}^{\circ}$ 1. DOI : 10.4000/vertigo. 12031

Pigeon P., 2015. Risque digue : une justification à la relecture systémique et géopolitique des risques environnementaux, L'Espace Politique, vol. 24, n 3. DOI : 10.4000/espacepolitique. 3256

Pitié C., Puech P., 2010. Expertise complémentaire des zones de solidarité délimitées en Vendée suite à la tempête Xynthia survenue dans la nuit du 27 au 28 février 2010, rapport $\mathrm{N}^{\circ}$ 007336-01, CGeDD / MEEDDM, 80 p.

SOGREAH, 2010. Éléments de mémoire sur la tempête Xynthia du 27 et 28 février 2010 en Charente-Maritime, Rapport No 4311608 -FLU/SEE, Préfecture de la Charente-Maritime / DDTM 17, $792 \mathrm{p}$.

Verger F., 2010. Xynthia, zones d'ombre sur les zones noires, Études Foncières, no 145, p. 6-9.

VINET F., 2010. Le risque inondation. Diagnostic et gestion. Éditions Lavoisier, Paris, 318 p.

Vinet F., Boissier L., Defossez S., 2011. La mortalité comme expression de la vulnérabilité humaine face aux catastrophes naturelles: deux inondations récentes en France (Xynthia, Var, 2010), VertigO - la revue électronique en sciences de l'environnement, vol. 11, n 28. DOI : 10.4000/ vertigo. 11074

Vinet F., Defossez S., Rey T., Boissier L., 2012. Le processus de production du risque « submersion marine » en zone littorale : l'exemple des territoires « Xynthia », Norois, n 222, p. 11-26. DOI : 10.4000/norois.3834 